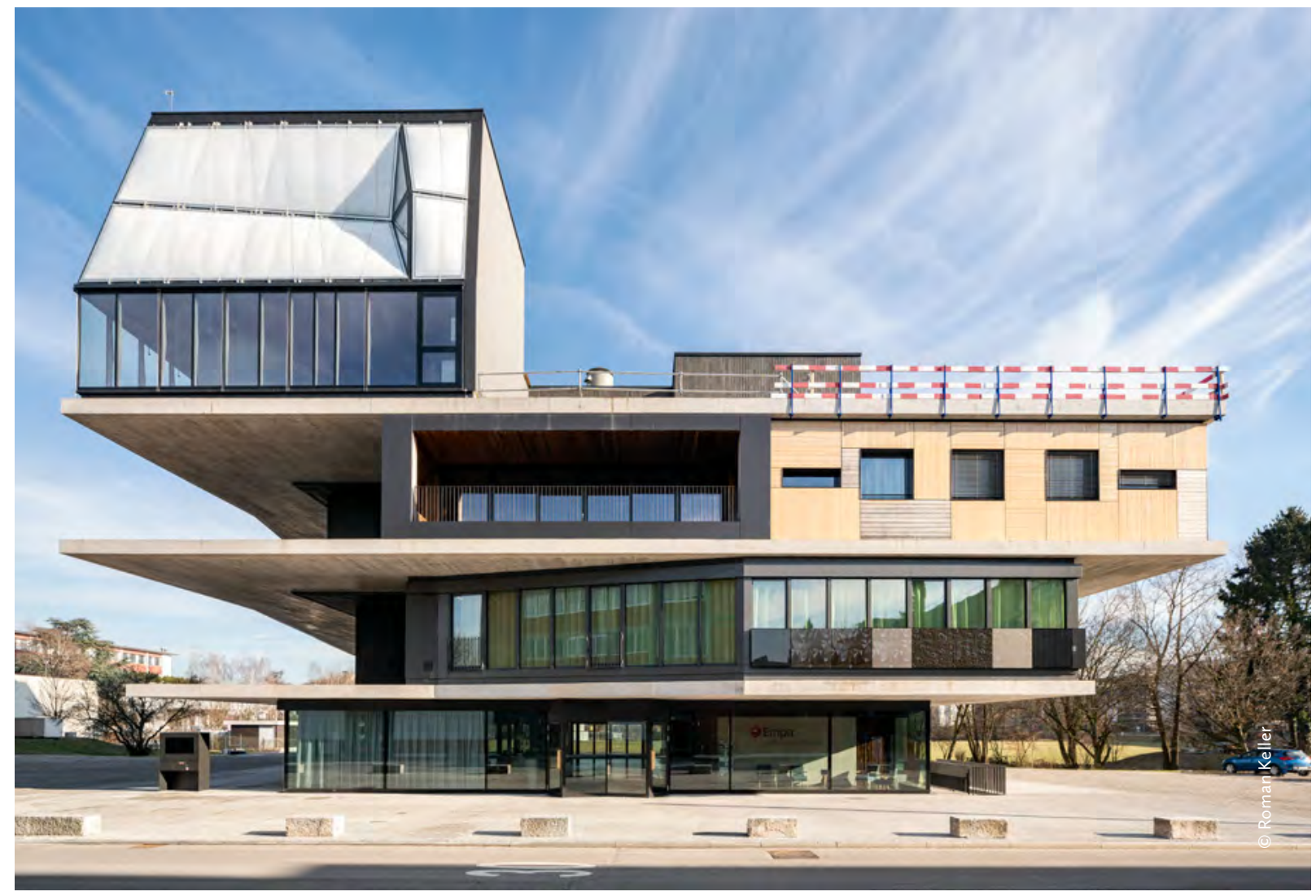

NEST

\title{
Edificio de investigación y residencia para Empa
}

Palabras clave Especulación Edificio

Procesos Sustentabilidad Proyecto
En tanto combina experimentación material e investigación académica, un centro para el desarrollo de tecnologías innovadoras es la plataforma ideal para la especulación arquitectónica. Partiendo de esta idea, el siguiente edificio muestra cómo la arquitectura, entendida como infraestructura, es capaz de proveer las condiciones para reunir a la industria y la academia en un mismo ámbito de especulación. 


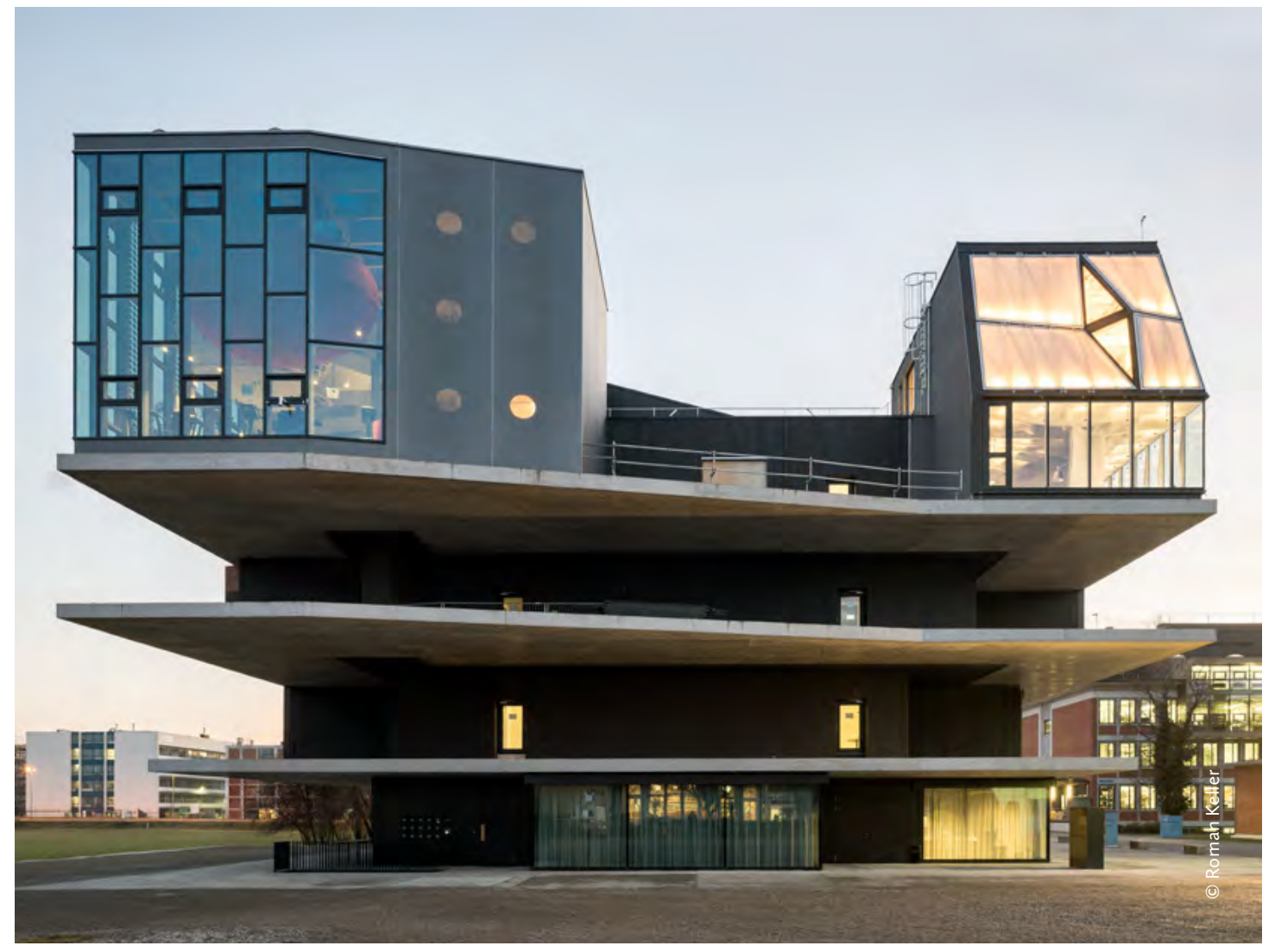

GRAMAZIO KOHLER

ARCHITECTS

P

Dübendorf, Suiza

2016

a reducción de la huella ambiental de los edificios es uno de los principales desafíos que enfrenta hoy la industria de la construcción. Para ello, se desarrolla una investigación intensiva tanto en el mundo académico como en el de la industria, que abarca todos los niveles: desde los materiales hasta los sistemas constructivos y el funcionamiento de los edificios. Debe hacerse especial hincapié en el problema de la transferencia de tecnología, ya que el sector de la construcción es conocido por su lenta incorporación de innovaciones. Además, generalmente se omite la interacción del usuario con soluciones recién 


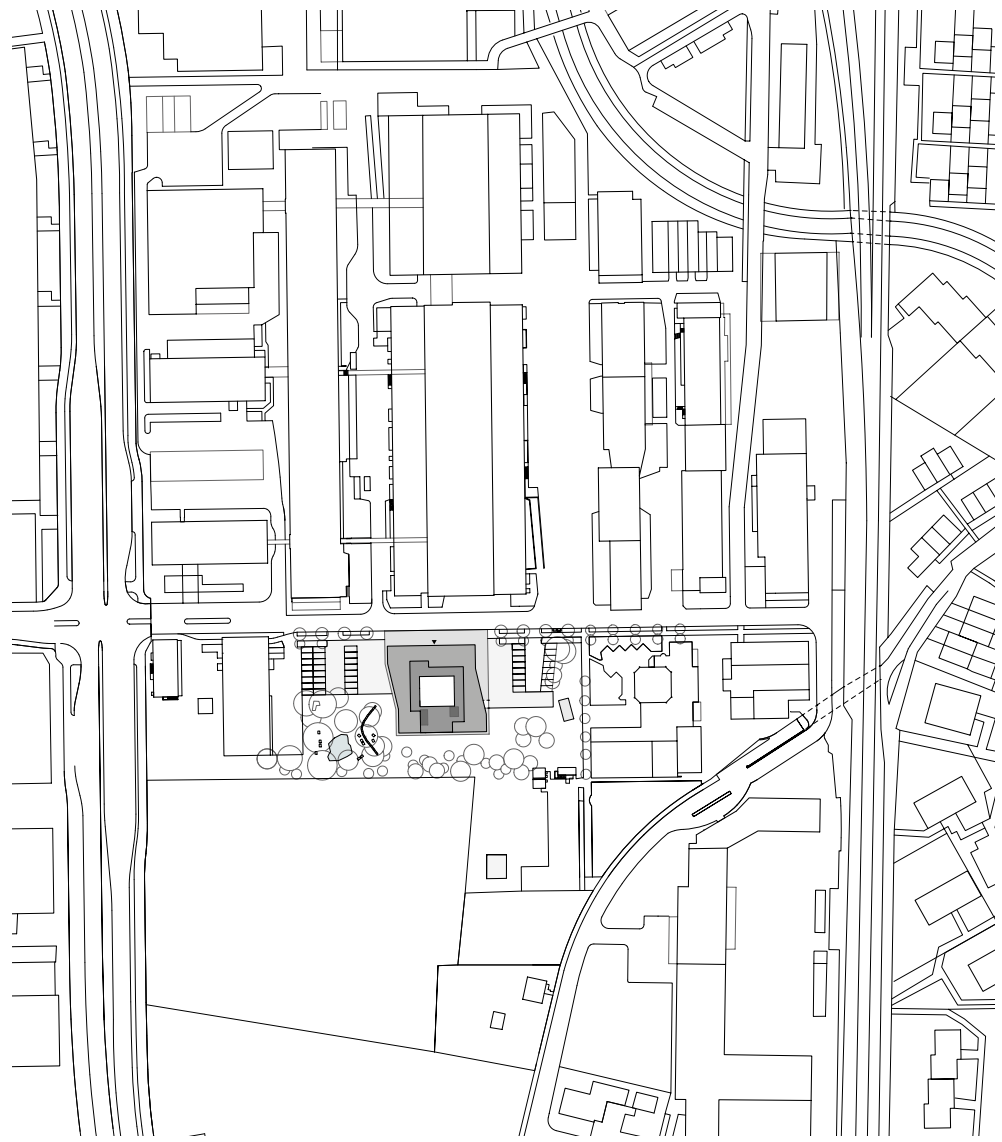

desarrolladas durante la fase de investigación, pero este juega un papel clave para su aceptación en el mercado.

NEST aborda estos problemas desde un enfoque holístico: es una plataforma modular y dinámica de investigación y demostración para tecnologías de construcción avanzadas e innovadoras, ubicada en el corazón del campus Empa/Eawag, los laboratorios federales suizos para la ciencia y la tecnología de los materiales, y la investigación sobre el agua, respectivamente. Utilizado como residencia académica y espacio de trabajo experimental, Nest funciona como un laboratorio viviente para la construcción sustentable que permite desarrollar, testear y optimizar materiales, componentes y sistemas innovadores en condiciones reales. Nest consiste en una 'columna vertebral' central que funciona como estructura portante y punto de acceso a servicios y redes, junto a una grilla básica donde se ubican las cerca de 50 unidades de oficina o vivienda. Gracias a su diseño altamente flexible, que permite el intercambio de unidades de vivienda/trabajo o incluso pisos completos en modo plug-and-play, NEST cambiará constantemente su imagen abordando los temas 'candentes' de cada momento.

NEST se utilizará para concursos internacionales competitivos, lo que permitirá atraer las ideas más brillantes y las nociones experimentales más 

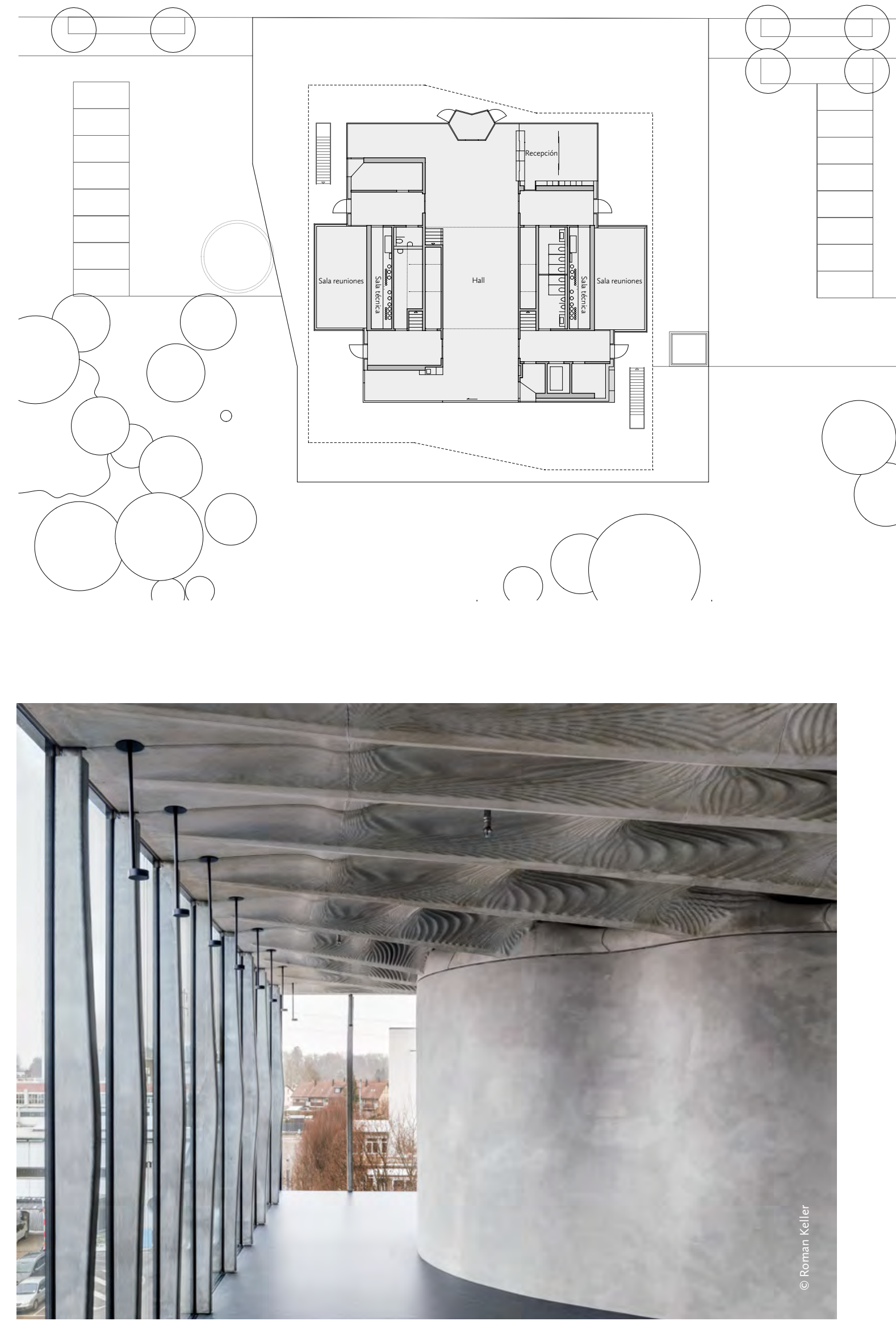


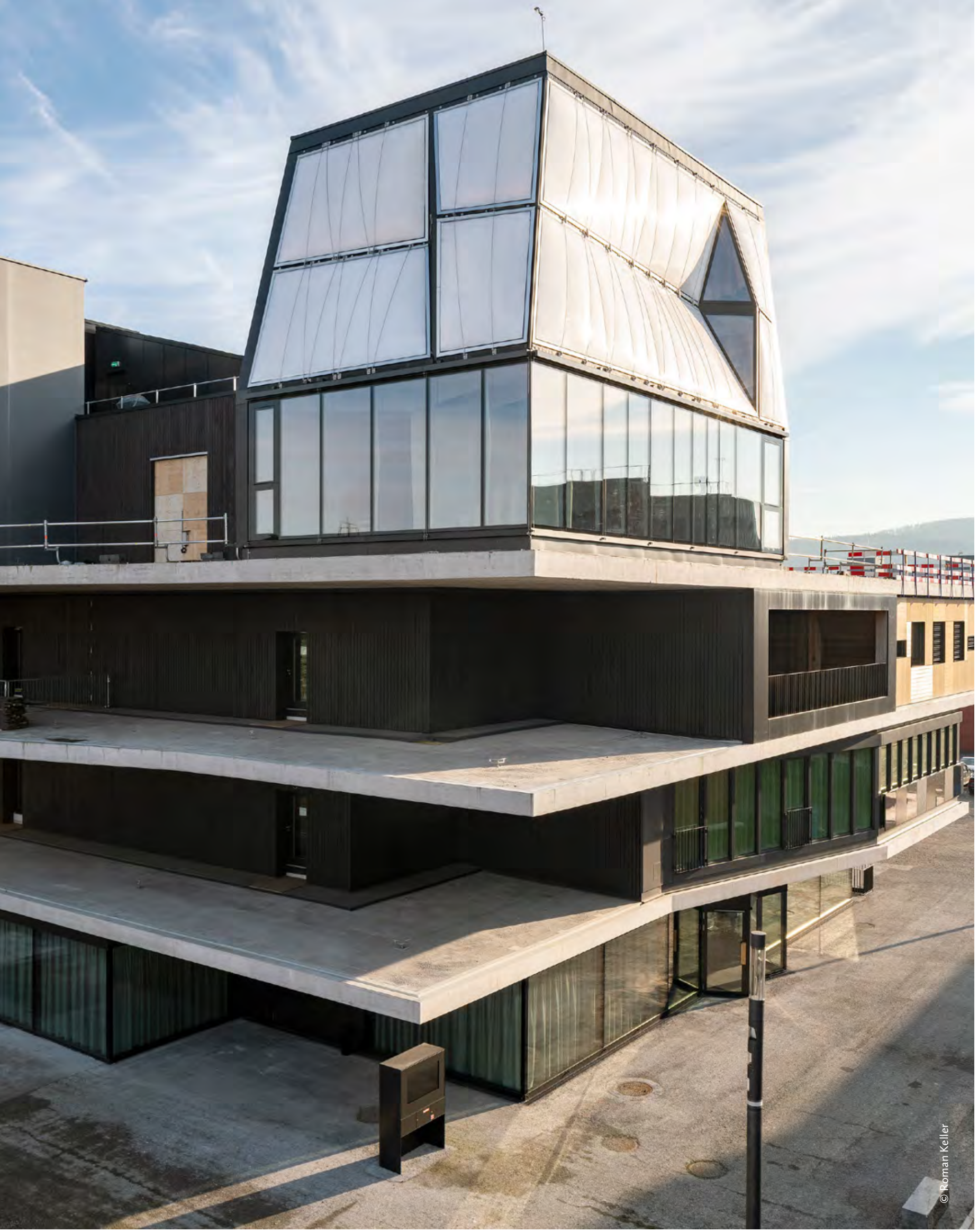


Planta segundo nivel / Second level plan

E. / S. 1: 500

$\theta$

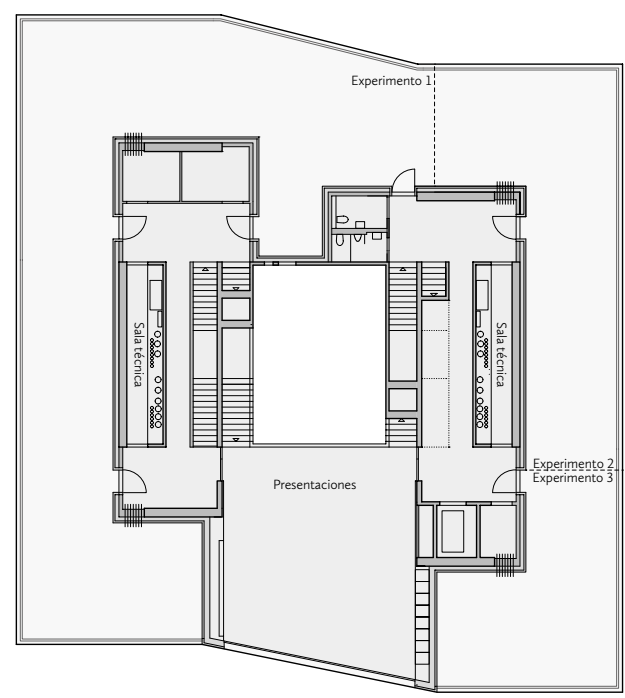

Planta cuarto nivel / Fourth level plan

E. / S. 1: 500

$\theta$

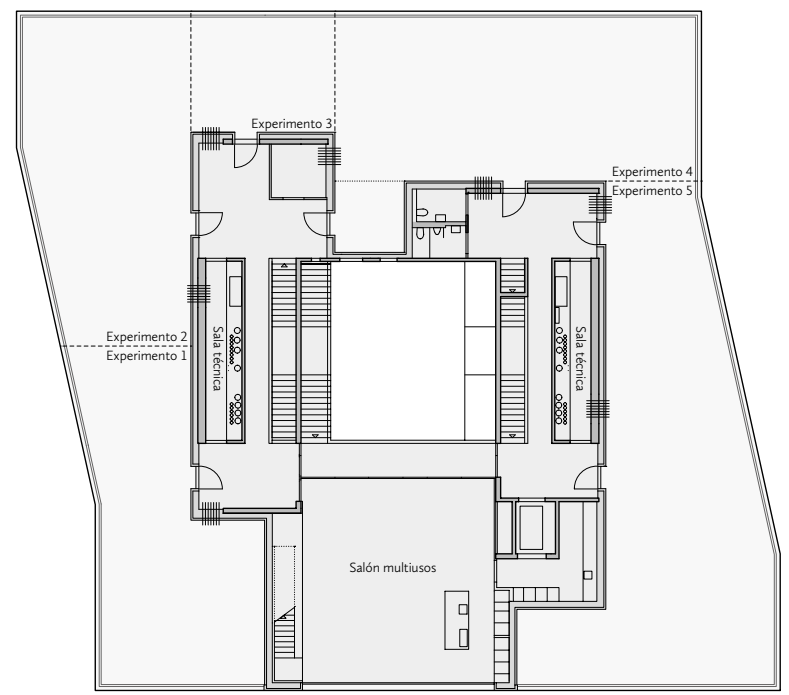

Planta tercer nivel / Third level plan

E. / S. 1: 500

$\theta$

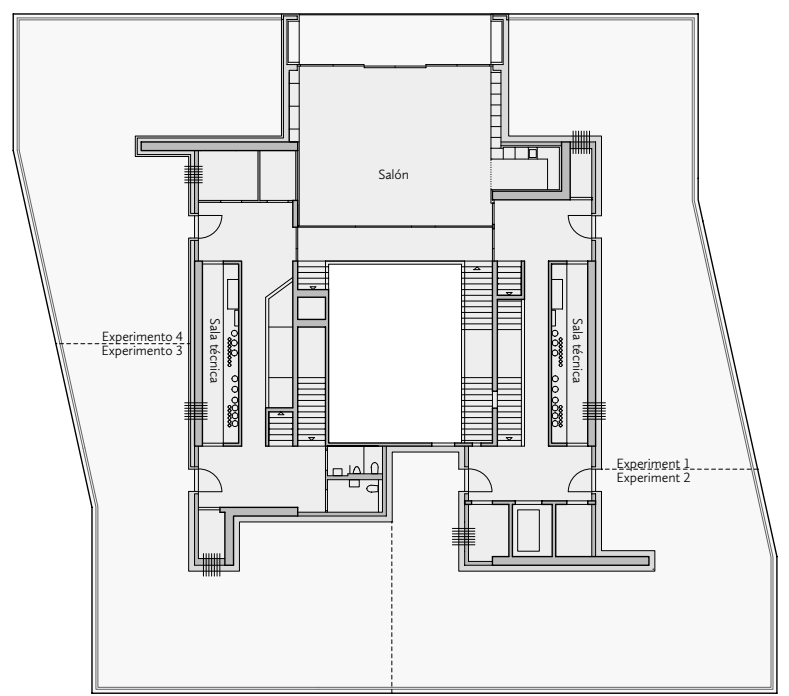

Planta techo terraza / Roof terrace plan

E. / S. 1: 500

$\theta$

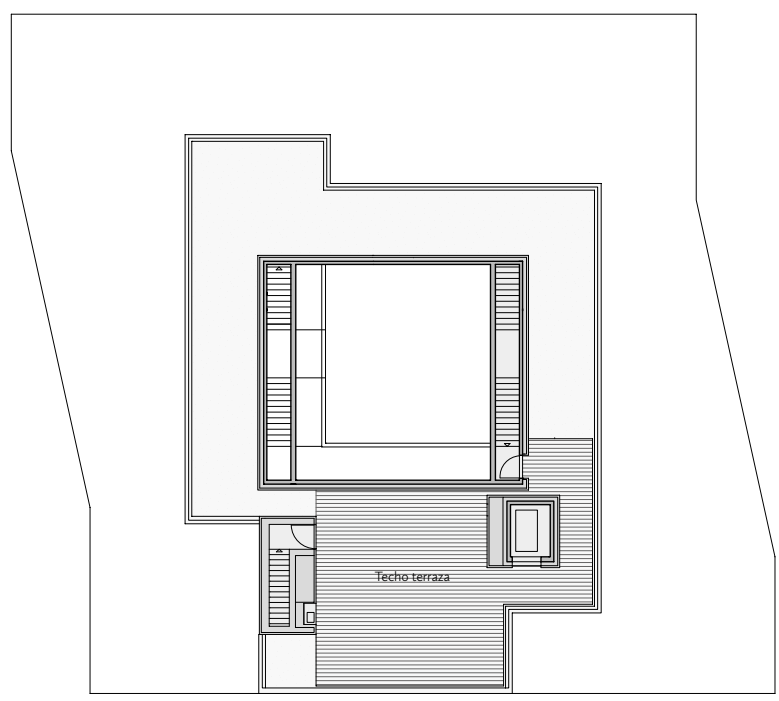


Elevación norte / North elevation E. / S. 1: 500

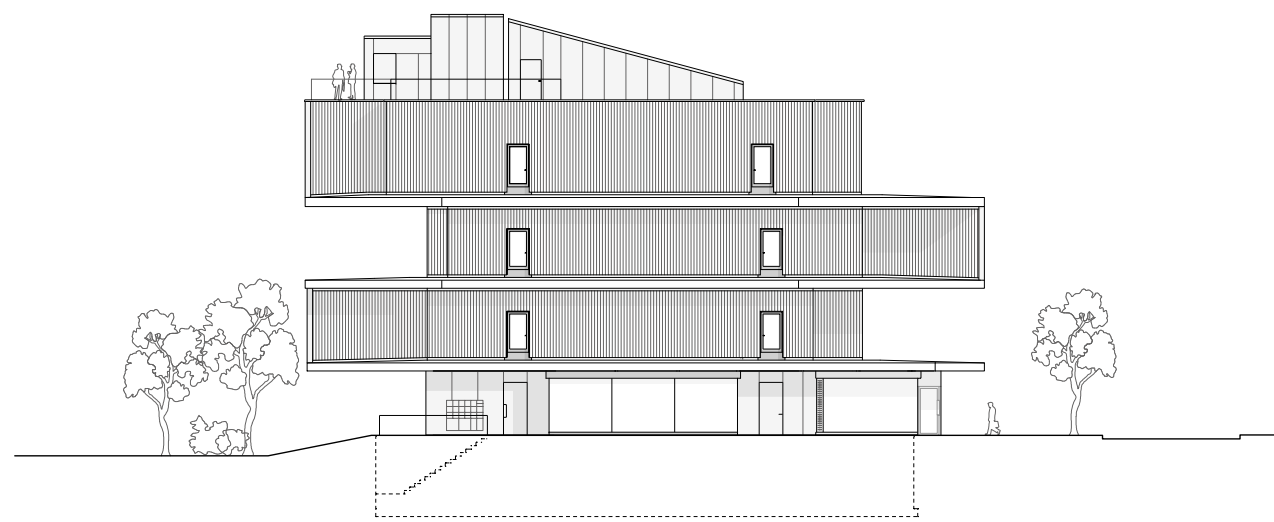

Elevación poniente / West elevation

E. / S. 1: 500

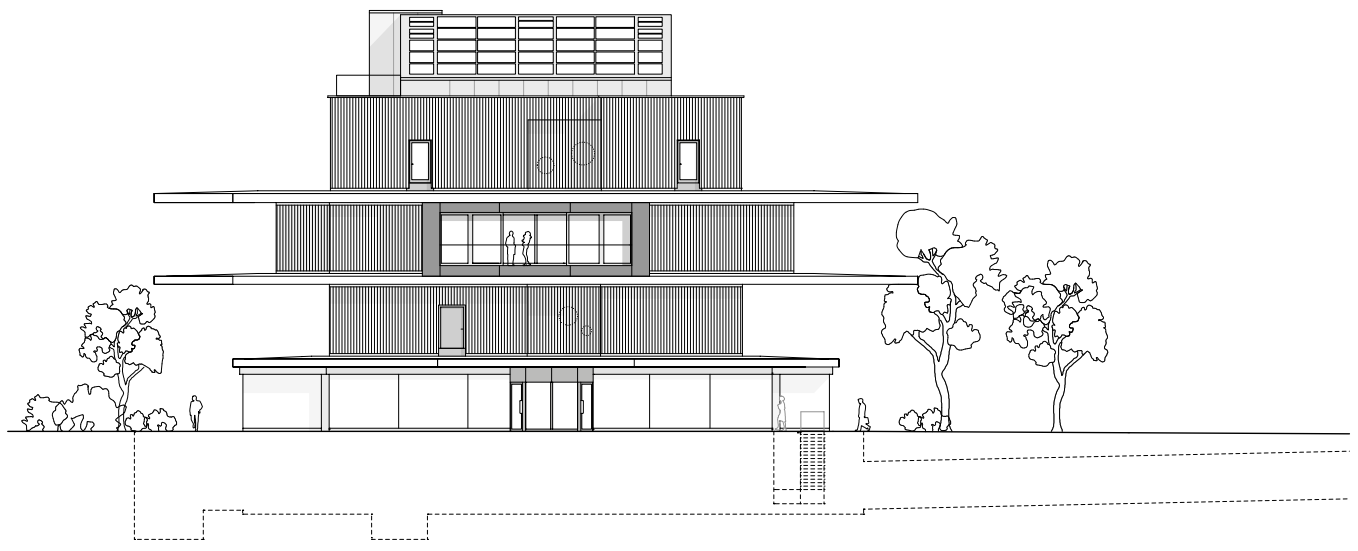



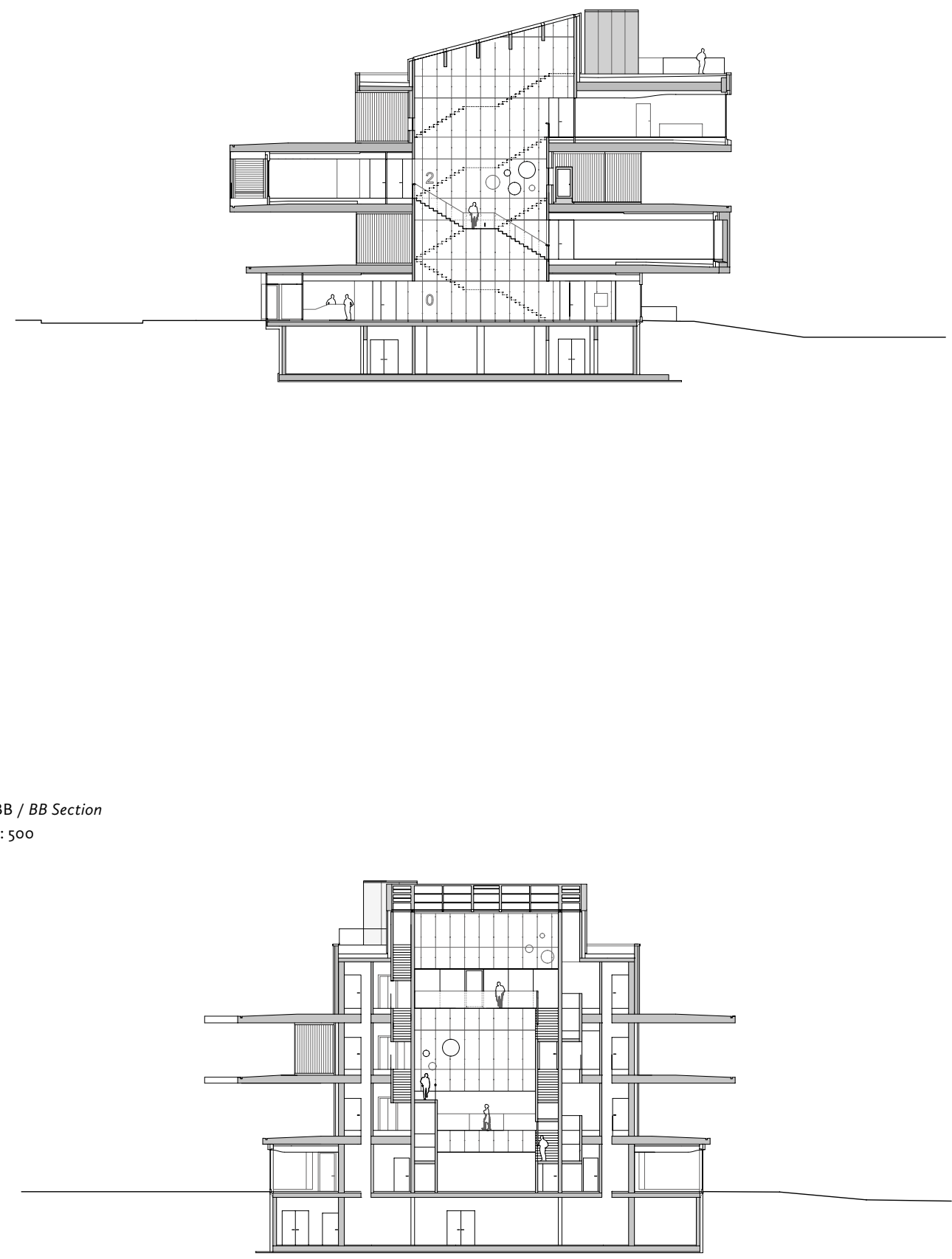


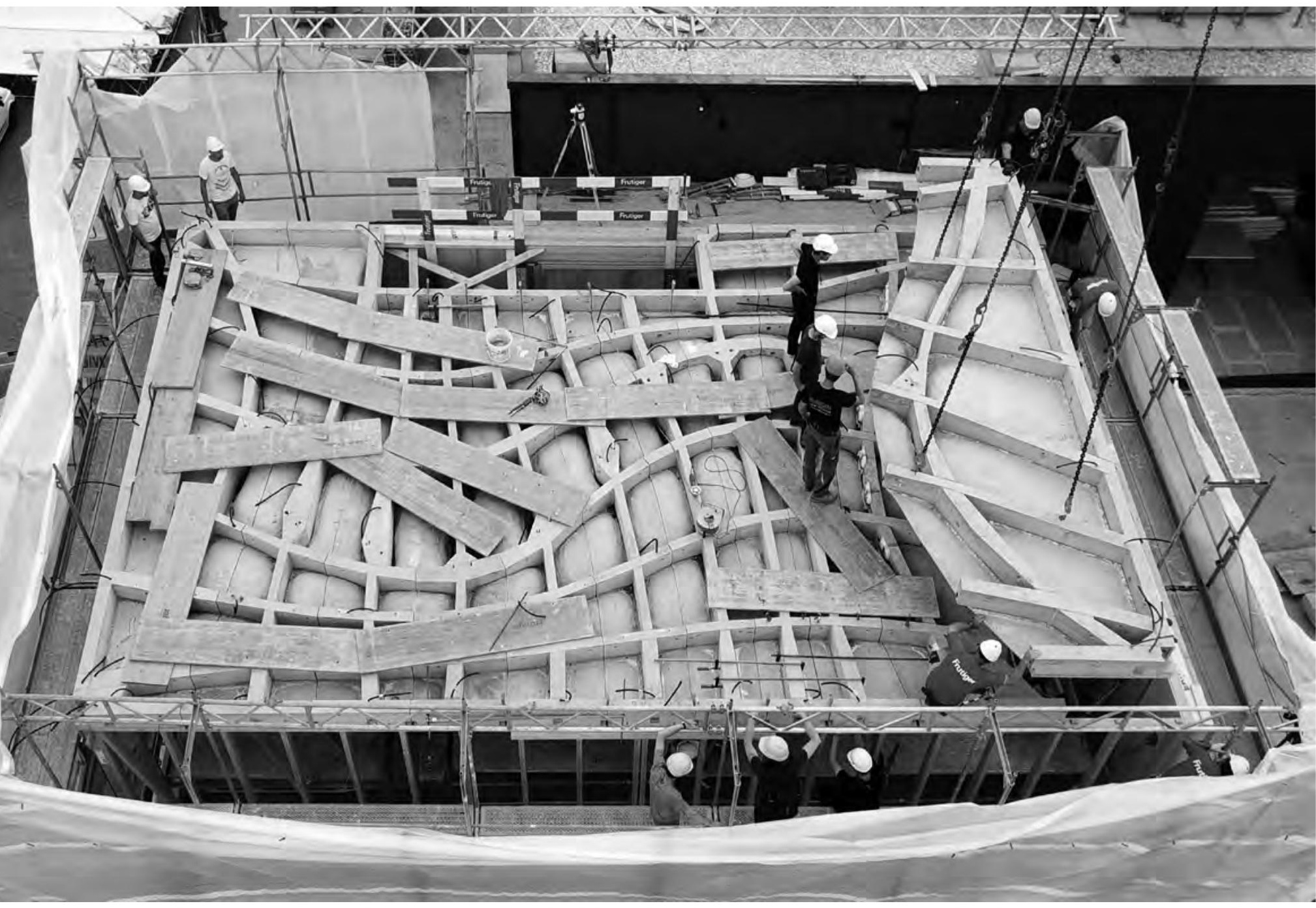

Instalación de losa inteligente / Smart slab installation

(c) Digital Building

Technologies (D BT), ETH Zürich / Tom Mundy avanzadas de universidades, centros de investigación e industrias. El objetivo es impulsar la colaboración internacional y la innovación en el sector de la construcción. Los problemas emergentes serán evaluados en estrecha colaboración con la industria de la construcción y se abrirán convocatorias para cada tema de investigación, de forma tal de seleccionar los proyectos más 'vanguardistas' tanto de la academia como de la industria a nivel internacional. Cada una de estas 'encarnaciones' de NEST irá acompañada de una serie de conferencias, seminarios, exposiciones, cursos de educación continua y clases abiertas. Como proyecto de investigación colectiva de la ETH, NEST cuenta con el apoyo de Empa, Eawag, Е т н Zurich y EPF Lausanne. Las instituciones asociadas forman una red interdisciplinaria con grupos de investigación en ciencia de materiales, ingeniería, arquitectura y ciencias sociales. La plataforma también estará abierta a colaboradores de la industria para apoyar el desarrollo de nuevas tecnologías y sistemas. ARQ 


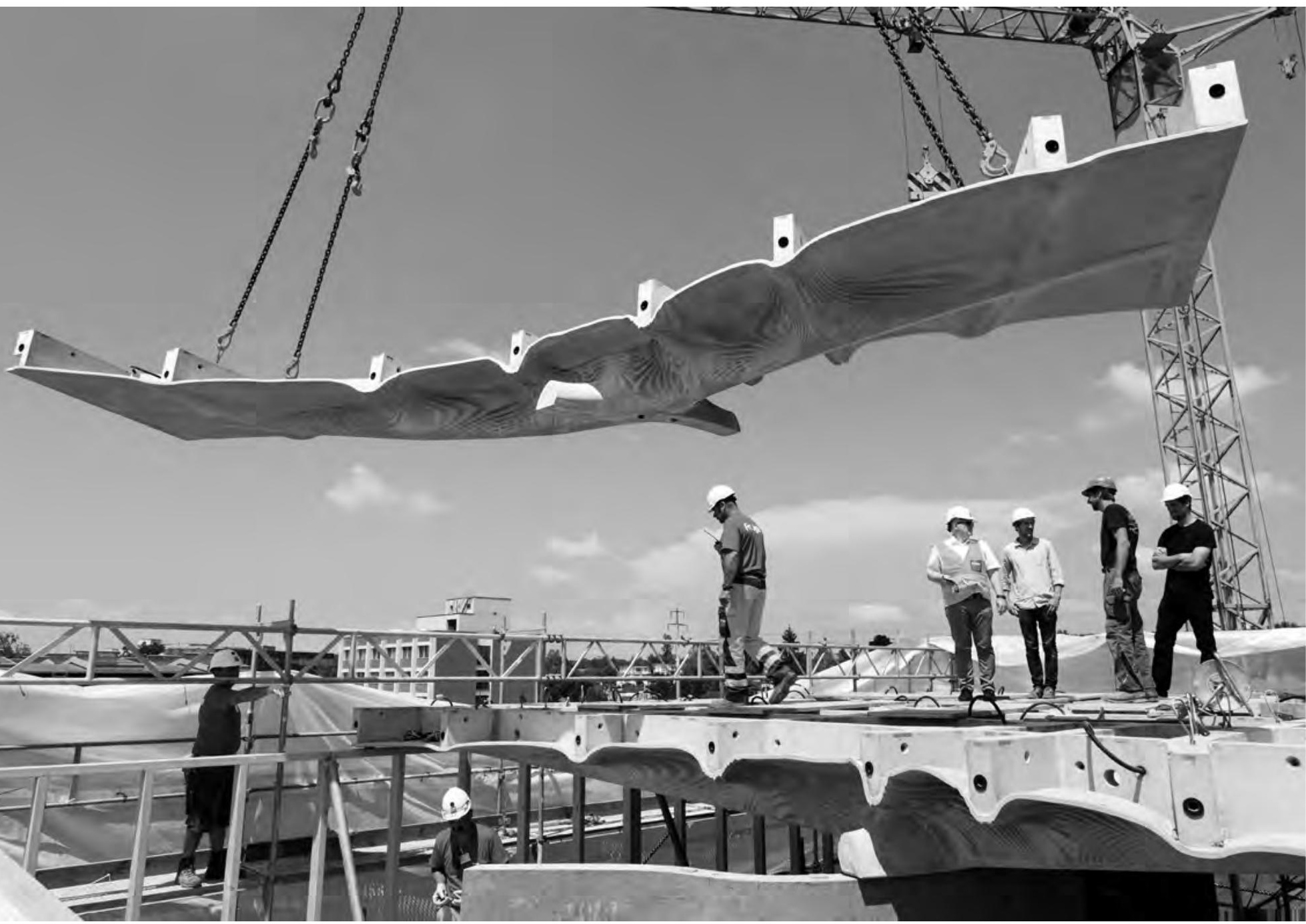

NEST

Arquitectos / Architects: Gramazio Kohler Architects, Zurich Colaboradores / Collaborators: Philipp Hübner, Claudia Kuhn, Sarah Schneider, Julian Gatterer, Matthias Helmreich, Kathrin Hiebler, Marco Jacomella, Panagiota Michailidou, Jürgen Pauger, Marion Ott, Sebastian Pajakowski, Poltak Pandjaitan, Henning Proske, Christian Schwarzwimmer, Miriam Zehnder, Basile Diem Ubicación / Location: Überlandstrasse 129 8600, Dübendorf, Switzerland
Cliente / Client: Empa Materials Science \& Technology, Dübendorf Materiales / Materials: Hormigón armado / Reinforced concrete Presupuesto / Budget: us $\$ 3500 / \mathrm{m}^{2}$ Superficie construida / Built area: $4935 \mathrm{~m}^{2}$ Superficie terreno / Site area: $5795 \mathrm{~m}^{2}$ Año de proyecto / Project year: 2010 - 2014 Año de construcción / Construction year: 2014 - 2016

\section{Gramazio Kohler Architects}

<info@gramaziokohler.com>

Con sede en Zúrich, la oficina combina en sus proyectos la dimensión material de la arquitectura construida con lógicas provenientes del mundo digital. Proyectan relaciones espaciales y comportamientos a través de la programación, utilizando el potencial de la computación y la fabricación digital de forma complementaria a los métodos tradicionales de diseño y construcción. 